\title{
Analysis of Participation and Performance in Half Marathon Runners
}

\author{
Ana Paula Sierra ${ }^{1,2,3}$, Marino Benetti ${ }^{1}$, Nabil Ghorayeb ${ }^{2}$, Carlos Sierra ${ }^{2}$, Flavia da Cunha Bastos ${ }^{1}$, Ary Rocco \\ Junior $^{1,4}$ and Maria Augusta Peduti Dal Molin Kiss ${ }^{1}$ \\ 1. School of Physical Education and Sport, University of São Paulo, São Paulo, São Paulo 05508-030, Brazil \\ 2. Sports Cardiology Department, Dante Pazzanese Institute of Cardiology, São Paulo, São Paulo 04012-909, Brazil \\ 3. Department of Medicine, Nove de Julho University, São Paulo, São Paulo 02169-280, Brazil \\ 4. Sports Management Master Program, Nove de Julho University, São Paulo, São Paulo 01156-080, Brazil
}

\begin{abstract}
Endurance running became a mass phenomenon throughout the 1980s around the world. Therefore, we wanted to investigate the participation and performance trends of runners in a half marathon. We analyzed a database containing the number of people who signed up and finished as well as age group, gender, placement, and runtime for all runners from a half marathon between 2003 and 2009. More than 35,000 runners participated in the half marathon, with an $80 \%$ increase in total participation during the 7 years analyzed. We verified that the annual participation increased, with a greater increase seen in female and middle-aged people of both genders, and runtimes of last finishers increased gradually in most of the age groups and both genders. However, while the number of people who signed up increased, there was a decrease in the number of participants who finished resulting in a negative correlation. This finding in association with the decrease in the percentage of finishers shows that each year the fitness deteriorated in both genders and across all ages.
\end{abstract}

Key words: Endurance, lifestyle, sports management.

\section{Introduction}

In the past century, due to modern life habits, man has become more technologically dependent and sedentary contrary to his ancestors who walked approximately 20 to $40 \mathrm{~km}$ a day in search of livelihood [1]. An example is one study regarding of the health and physical activity in England, which revealed that the number of physically inactive people is growing in all age groups [2].

Despite, the importance of physical activity has been evidenced particularly for the benefits to psychosocial health, functional ability, overall quality of life, and reduction in functional losses associated with age $[2,3]$. In order to achieve an increase in physical activity, the main efforts are often focused on aerobic exercises

Corresponding author: Ana Paula Rennó Sierra, M.S., research fields: sports cardiology, sports medicine and sports management. E-mail: anasierra@usp.br. such as running and aerobic exercises.

The street races represent an alternative for a change of habits. Among the many reasons that motivate people to run a few kilometers stand out, such as concerns about one's health and body, the company of friends, accessibility and easy practice, low costs, seeking beauty standards, and even seeking unattainable performance goals $[1,4,5]$. Thus, endurance running became a mass phenomenon throughout the 1980s around the world. Typical endurance race distances range from $10 \mathrm{~km}$ to $100 \mathrm{~km}$ in ultra-marathons with most of the participants younger than 40 years. These long-distance races increased in popularity among people of all ages probably as a result of greater awareness, making participation in long-distance races part of an active lifestyle for thousands of people around the world with an increasing number of participants every year $[6,7]$.

The number of older participants, called "master 
athletes", have increased. Additionally, female participation must be taken into account mainly because this intense participation has already decreased differences in performance between males and females [8-10]. However, endurance events are associated with increase in medical events. To reduce the risk of the medical events during long-distance races, participants should be well prepared in terms of appropriate physical fitness $[11,12]$.

In Brazil, the growth phenomenon in the number of participants in street races has been observed, however, studies aiming to register and analyze participation from a gender and performance perspective are still rare mainly in Brazil [13]. The results in terms of this phenomenon in Brazil considering the run time how a performance, could generate knowledge to understand and manage better the risk of this kind of event. Therefore, the aim of the current study was to analyze the evolution in the participation of runners and changes in physical fitness as measured by runtime in the Corpore Half Marathon.

\section{Methods}

The Corpore Half Marathon is held by Corporea nonprofit organization founded in 1982. Since its inception, it has organized races in the city of São Paulo and is considered the largest running club in Latin America. The Corpore Half Marathon has taken place annually between the first and third Sunday of April in São Paulo, SP, since 2000. The data were obtained from an electronic database (www.corpore.org.br) containing the number of people who signed up and finished, an individual race identification number, age group, gender, placement and runtime for all runners participating in the Corpore Half Marathon between 2003 and 2009. The individual runtime registered in the event was automatically measured using a radio frequency identification chip system. The study was approved by the School of Physical Education and Sport Ethics Committee, University of São Paulo.
Concerning the period chosen, the data from 2003 was the first available data in the electronic database and the racetrack remained unchanged from 2003 to 2009. In 2010, the racetrack changed and the number of participants was restricted for the town hall of University of São Paulo, because the Corpore Half Marathon take place inside the campus and the main activity of the university is education and research.

To investigate the fitness, as measured by run time, the runners were separated into groups based on gender (M-male, F-female) and age. For example, 16-19 was used for runners between 16 and 19 years old, 20-24 was used for runners between 20 and 24 years old, and so on in 5-year intervals. Therefore, M16-19 corresponds to all male runners aged between 16 and 19 years old. For the purpose of analysis, the groups were considered overall, by gender, and by age group, which were analyzed for changes in minimum and maximum runtime, demonstrating the performance. All statistical analyzes were performed using SPSS software 19.0 (SPSS, Inc., USA, 2010) and macro in Microsoft Excel 2010 spreadsheet. The data analysis involved are presented as descriptive statistics, correlations between the variables, and linear regression to the runtime through the years to use the adjusted coefficient of determination $\left(r^{2}\right)$ to know if the variables have significant correlation.

\section{Results}

Between 2003 and 2009, a total of 36,999 people signed up for the Corpore Half Marathon and 32,595 finished the race (88.1\%) including 27,212 men (83.5\%) and 5,398 women (16.5\%), a ratio of 5.04/1.

According to Table 1, the number of people who signed up and finished in the Corpore Half Marathon increased every year from 4,078 and 3,641 in 2003 to 7,858 and 6,554 in 2009, respectively $\left(r^{2}=0.87, P<\right.$ 0.001 and $\left.r^{2}=0.9, P<0.001\right)$. As the number of people who signed up increased the percentage of finishers decreased from $89.28 \%$ in 2003 to $83.41 \%$ in $2009\left(r^{2}\right.$ $=0.56, P=0.05)$, resulting in a inverse correlation 
between the number of people who signed up and the percentage of finishers (correlation-0.91).

Table 2 shows that from 2003 to 2009 participation increased significantly overall by $80 \%$ with a $102.5 \%$ increase in female participation (589 to 1,193 finishers) and a $76.15 \%$ increase in male participation $(3,052$ to 5,376 finishers). We verified that the male/female ratio decreased from 5.18 to 4.50 during that period due to the growth in female participation, although the reduction was not significant $\left(r^{2}=0.41, P=0.11\right)$ (Table 2).

When analyzing participation growth by age group and gender (Fig. 1), we noted that there was larger growth in all age groups in both genders in percentages from 2006 to 2009. Furthermore, data shows that the age groups with the largest growth in finishers were 35-39, 40-44, and 45-49 from 2003 to 2009. However, the 16-19 and 20-24 age groups had a decrease in the number of finishers in the same period. This analysis shows a similar behavior, in perceptual, referring to growth in both genders.

As seen in Fig. 2A, the average runtime from 2003 to 2009 for the overall, female, and male winner was 01:04:31, 01:15:04, and 01:04:31, respectively $(P<$ 0.001). Additionally, as seen in Fig. 2A, the time of the first overall finisher refers to the time of the first male finisher, which has not changed much in the analyzed period from 01:04:50 in 2003 to 01:05:06 in 2009 $\left(r^{2}=\right.$ $0.02, P=0.74)$. However, the times of the first female finisher showed a large variation from 01:17:16 in 2003 to 01:11:41 in 2009, showing a reduction in runtimes through the years analyzed $\left(r^{2}=0.72, P=\right.$ 0.01).

Additionally, the runtime of the last overall finisher changed among men and women (Fig. 2B). We observed that in 2004 there was an increasing trend in the completion time of the last finisher for both female and male runners $\left(r^{2}<0.5, P>0.05\right)$.

Table 1 Number of participants signed up, finishers, and percentage of starters in the Corpore Half Marathon from 2003 to 2009.

\begin{tabular}{llll}
\hline Year & Signed up & Finishers & $\%$ \\
\hline 2003 & 4078 & 3641 & 89.28 \\
2004 & 4086 & 3694 & 90.41 \\
2005 & 4465 & 4102 & 91.87 \\
2006 & 4664 & 4255 & 91.23 \\
2007 & 5611 & 5001 & 89.13 \\
2008 & 6237 & 5348 & 85.75 \\
2009 & 7858 & 6554 & 83.41 \\
Total & 36999 & 32595 & 88.10 \\
\hline
\end{tabular}

Results are show in absolute numbers or percentual. \%: percentage of starters who finished the race.

Table 2 Total, female, and male finishers and M/F ratio in the Corpore Half Marathon from 2003 to 2009.

\begin{tabular}{lllll}
\hline Year & Total & Female & Male & M/F Ratio \\
\hline 2003 & 3641 & 589 & 3052 & 5.18 \\
2004 & $3694(1.45 \%)$ & $583(-1.02 \%)$ & $3111(1.93 \%)$ & 5.33 \\
2005 & $4102(11.05 \%)$ & $655(12.34 \%)$ & $3447(10.80 \%)$ & 5.26 \\
2006 & $4255(3.73 \%)$ & $636(-2.90 \%)$ & $3619(4.99 \%)$ & 5.69 \\
2007 & $5001(17.53 \%)$ & $849(33.49 \%)$ & $4152(14.72 \%)$ & 4.89 \\
2008 & $5348(6.94 \%)$ & $893(5.18 \%)$ & $4455(7.30 \%)$ & 4.98 \\
2009 & $6554(22.50 \%)$ & $1193(33.59 \%)$ & $5376(20.67 \%)$ & 4.5 \\
Annual growth & $485.5(10.54 \%)$ & $242.2(13.45 \%)$ & $387.3(10.07 \%)$ & \\
20032009 & $2913(80 \%)$ & $1453(102.5 \%)$ & $2324(76.15 \%)$ & \\
\hline
\end{tabular}

Results are shown in absolute numbers (percentage growth over the previous year). Annual growth: the average annual growth from 2003 to 2009; 2003-2009: growth in the number of participants from 2003 to 2009. 

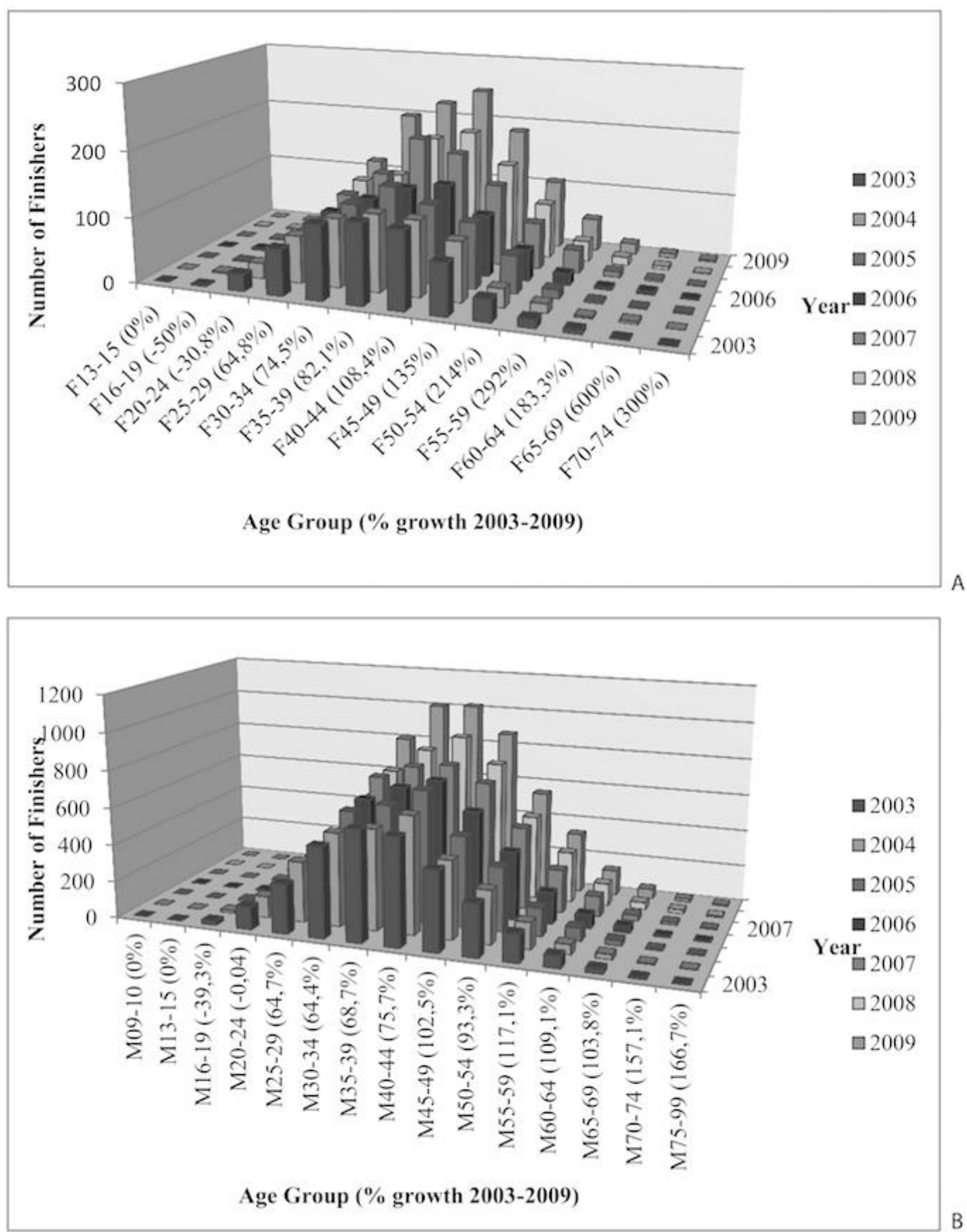

Fig. 1 Number of finishers per age group and percent growth from 2003 to 2009 in the Corpore Half Marathon. For definitions of the age groups see methods.

When age groups were analyzed (Fig. 3), we found a decrease in the completion time for the first finisher in the age groups F45-49 and M50-54 $\left(r^{2}=0.68\right.$ and 0.5, respectively, $P \leq 0.05$ ); however, an increase in time for the first finisher was observed for the age groups F70-74 and M65-69 $\left(r^{2}=0.76\right.$ and 0.7, respectively, $P$ $\leq 0.05$ ). The times remained unchanged for the other age groups. An increasing trend was found for the age groups F16-19, F20-24, F40-44, F50-54, F60-64, F65-69, M16-19, M20-24, and M40-44. As shown in Fig. 3, the same profile was observed throughout the years regarding the evolution of runtimes for both genders in the age groups 16-19, 20-24, 25-29, 30-34, and 40-44. 

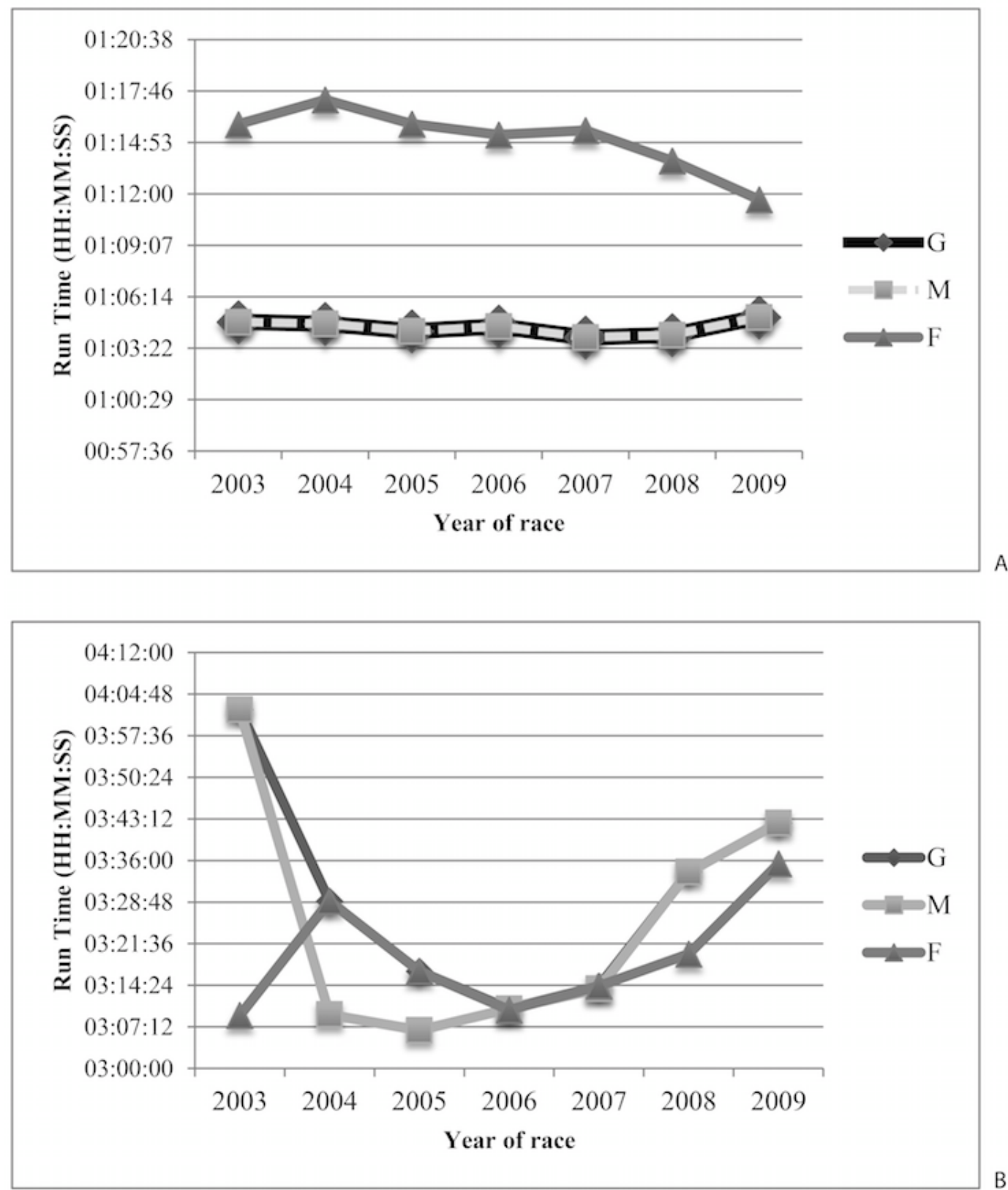

Fig. 2 Performance measured by race completion time of the first finisher (A) and last finisher (B) in the overall (G), male (M), and female (F) groupin the Corpore Half Marathon from 2003 to 2009.

Concerning the results of the runtimes of the last finishers (Fig. 4), we found a decreasing trend in the runtime of the age group M70-74, while the runtimes remained stable for the age groups F20-24, F30-34, F60-64, M35-39, and M65-69. In the age groups F35-39, F50-54, F55-59, F70-74, and M50-54 $\left(r^{2}=\right.$ $0.94,0.69,0.86,0.67,0.53$, respectively, $P \leq 0.05$ ), there was an increase in the runtime of the last finisher between 2003 and 2009. In all the other age groups, there was an increasing trend in the time of the last finisher. We did not find a correlation between variables in the analysis of race times of the last finisher and the number of finishers.

\section{Discussion}

In the present study, we evaluated some characteristics related to performance of the finishers of Corpore Half Marathon with more than 35,000 runners 

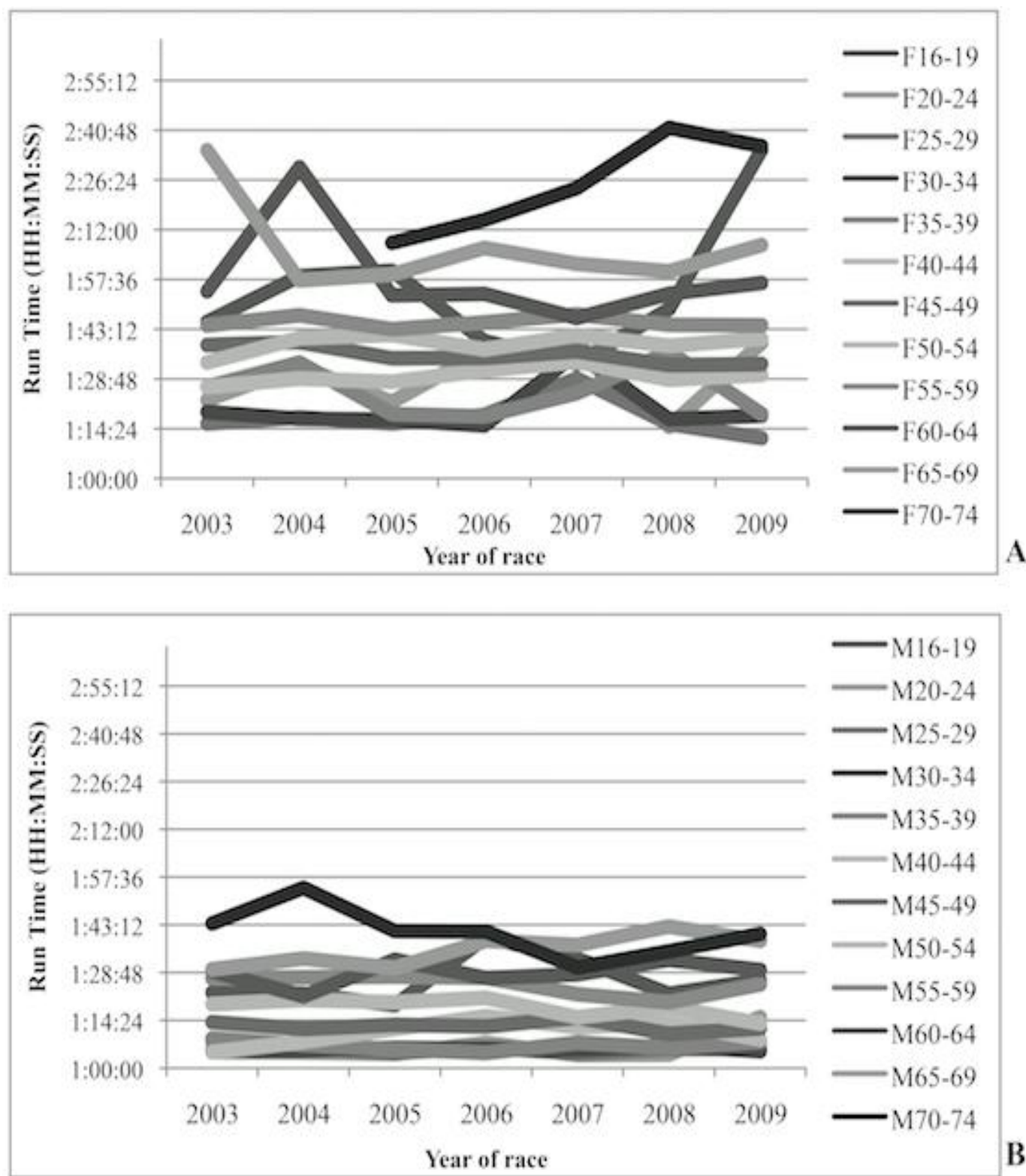

Fig. 3 Performance measured by race completion time of the first female finisher (A) and first male finisher (B) per age group in the Corpore Half Marathon from 2003 to 2009. For definitions of the age groups see methods.

for a period of seven years. We were able to verify that the annual participation increased, with a greater increase in female participants and middle-aged people of both genders, but also that the race completion times of the last finishers have increased gradually in most of the age groups analyzed and for both genders. With regard to runtimes for the first finishers, there was a significant decrease for female runners, while the time remained stable for the male runners. However, while the number of participants who signed up increased, there was a decrease in the number of finishers, resulting in a negative correlation. Overall, our results show a profile of growth in the number of participants who are less physically prepared for running the half marathon, which is reflected in the increase in completion times of the last finishers and lower percentage of finishers. This phenomenon is opposite to what is observed with elite athletes who have been maintaining or reducing their runtimes.

The findings of the present study concerning the 

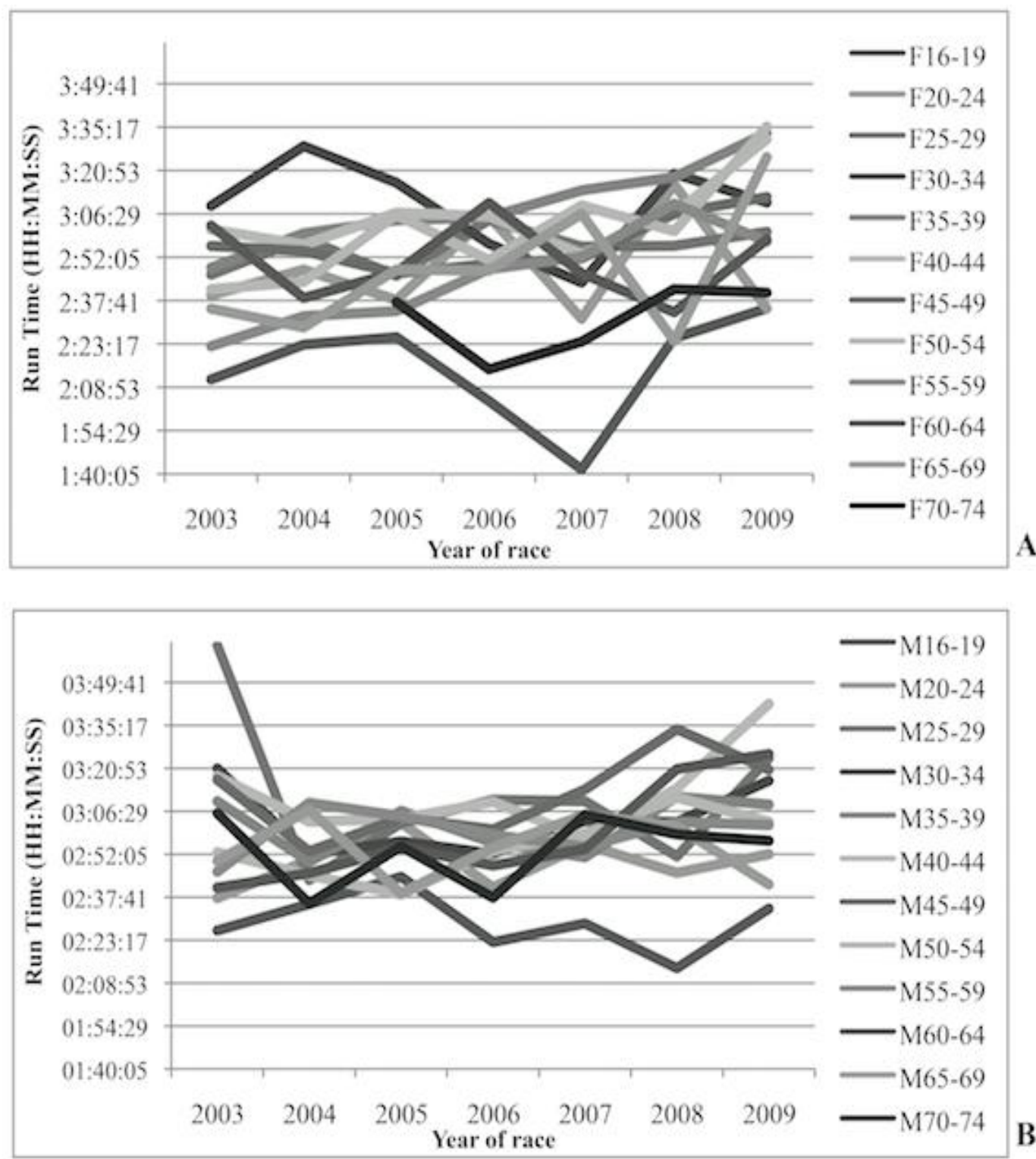

Fig. 4 Performance measured by race completion time of the last female finisher (A) and last male finisher (B) per age group in the Corpore Half Marathon from 2003 to 2009. For definitions of the age groups see methods.

increase in participation of the half marathon, particularly that of middle-aged and older individuals as well as the change in the proportion of male and female participants, is consistent with other studies that have analyzed participation in marathons, ultramarathons, and Ironman competitions [6, 7, 14-16] and seen growth rates in seven years equivalent to or higher than the rates shown in studies that have analyzed periods of 10 to 17 years of growth. The increase in popularity of endurance and ultra-endurance sports is related to a growing number of participants all over the world and not only due to the positive effects of life expectancy and health in general, which can be reflected in the increased number of participants in the older age groups $[14,15,17,18]$.

The large decrease in the runtimes for the first female finishers also corroborates findings of previous studies [19], showing that women are achieving improvements in their performances. This is due to the fact that the insertion of women in sports occurred only in the mid-nineteenth century, and thanks to their inclusion participation in the first decades of the 
twentieth century women have achieved more [9]. However, women's runtimes still remain higher than their male counterparts, which was demonstrated when we observed that women still need $10.1 \%$ more time than men to complete the half marathon. Additionally, regarding female participation, the decrease in the male/female ratio of 5.18 to 4.50 corroborates the results found by many researchers who also demonstrated a greater decrease in this ratio (2.47 in 1999 in the New York Marathon and 2.60 in the Germany Half Marathon) [16]. This shows that the female participation is still way lower than that of the male participants, and lower than female participation in other countries despite growth in Brazil $[16,19]$.

In the present study, we observed a larger growth in the number of participants between the ages of 35 and 49 , the same age groups found in many studies [7, 19]. However, other studies found that a larger number of runners were in the category of 30 - to 45 -year-olds and others greater participation between the ages of 45 and $59[5,13]$. According to the data of the FPA (São Paulo Athletics Federation), 70\% of the participants in street races are 40 years old and older $[1,5,13]$. The increase in these age groups may be related to the fact that older adults identify the benefits of physical activity in reducing the effects of age and maintaining functional capacity for daily and family activities in addition to the mental health benefits such as the feeling of relaxation, reduced anxiety, and improved humor [2, 20]. However, the decrease in participation of the 16to 24-year-olds that we found may be due to the fact that many young women at this age are more concerned with impressing their boyfriends, are worried about appearing less feminine, and mainly because this age group seems to be bored by the practice of this type of activity [2, 21].

With regard to the times of the first finishers, the increase in completion times for most female age groups and male participants for the age groups of 16-19, 20-24, 40-44 and 65-69, has previously been observed in the Spartathlon ultramarathon, but differs from the data presented by Lepers and Cottagni [7, 18]. The runtimes of the last finishers only showed a tendency towards improvement for the age group M70-74, reflecting the previously unexplored potential. A decrease or maintenance of performance for the last finishers occurred in all other age groups, suggesting that the participants are less prepared compared with those who participated seven years ago. Also, the increase in the number of participants refers to individuals who are not athletes and have varied personal interests $[1,6]$.

We acknowledge several limitations in our study. For example, for the analysis of racetimes, the climate factor was not taken into consideration though it is known to affect performance; however, the race always occurs the same time of year making this variable a less important limitation. Another limitation refers to the period analyzed because the USP limited the number of participants in those events to 6,000 people in 2010 .

\section{Conclusion}

The evaluation of performance trends and evolution of the number of participants in the Corpore Half Marathon from 2003 to 2009 leads us to the following some conclusions. Firstly, the growth in participation of the Corpore Half Marathon in the period analyzed was observed indicating thereby that there is a trend towards greater engagement of people in activities designed to improve physical fitness. Secondly, the increase in participation is associated with an increase in completion times of the race and decrease in the percentage of finishers which shows that each year the participants are less prepared for this kind of race, with a gradual increase in the risk related to their participation.

These results lead us to conclude that only the participation in racing events does not mean that the benefits of the race for health practice happen. Therefore, we emphasize the importance of performing clinical tests and cardiovascular evaluations, and providing proper guidance regarding physical activities 
to ensure the good health of the individual. Such information could reveal which aspects of the preparation of the participants are being neglected. Additional studies are necessary to provide better understanding of the street race growth phenomenon as well as how it relates to performance variables.

\section{Acknowledgements}

Thanks to TEB (BrazilianElectronic Technology) for support to this project.

\section{References}

[1] Salgado, J. V. V., and Chacon-Mikahil, M. 2006. "Street Race: Analyses of the Growth of the Number of Competitions and Practioners." Conexões 4 (1): 90-9. (in Portuguese)

[2] Allender, S., Cowburn, G., and Foster, C. 2006. "Understanding Participation in Sport and Physical Activity among Children and Adults: A Review of Qualitative Studies." Health Education Research 21 (6): 826-35.

[3] Wiswell, R. A., Hawkins, S. A., Jaque, S. V., Hyslop, D., Constantino, N., Tarpenning, K., Marcell, T., and Schroeder, E. T. 2001. "Relationship between Physiological Loss, Performance Decrement, and Age in Master Athletes." The Journals of Gerontology Series A: Biological Sciences and Medical Sciences 56 (10): M618-26.

[4] Truccolo, A. B., Maduro, P. A., and Feijó, E. A. 2008. "Motivational Factors of Adherence to Running Groups." Motriz 14 (2): 108-14. (in Portuguese)

[5] Silva, M. d. S., and Sousa, M. d. S. 2013. "O Papel Das Assessorias Esportivas No Crescimento Das Corridas De Rua No Brasil." FIEP Bulletin On-line 83 (1).

[6] Aagaard, P., Sahlen, A., and Braunschweig, F. 2012. "Performance Trends and Cardiac Biomarkers in a 30-Km Cross-Country Race, 1993-2007." Medicine Science in Sports and Exercise 44 (5): 894-9.

[7] Zingg, M. A., Knechtle, B., Rust, C. A., Rosemann, T., and Lepers, R. 2013. "Analysis of Participation and Performance in Athletes by Age Group in Ultramarathons of More Than $200 \mathrm{Km}$ in Length." International Journal of General Medicine 6: 209-20.

[8] Burfoot, A. 2007. "The History of the Marathon : 1976-Present." Sports Medicine 37 (4-5): 284-7.

[9] Goellner, S. V. 2006. "They Make History Between Incentives and Hindrances." Pensar a prática 8 (1): 85-100. (in Portuguese)

[10] Tanaka, H., and Seals, D. R. 2008. "Endurance Exercise
Performance in Masters Athletes: Age-Associated Changes and Underlying Physiological Mechanisms." The Journal of Physiology 586 (1): 55-63.

[11] Borjesson, M., Urhausen, A., Kouidi, E., Dugmore, D., Sharma, S., Halle, M., Heidbuchel, H., Bjornstad, H. H., Gielen, S., Mezzani, A., Corrado, D., Pelliccia, A., and Vanhees, L. 2011. "Cardiovascular Evaluation of Middle-Aged/ Senior Individuals Engaged in Leisure-Time Sport Activities: Position Stand from the Sections of Exercise Physiology and Sports Cardiology of the European Association of Cardiovascular Prevention and Rehabilitation." European Journal of Cardiovascular Prevention \& Rehabilitation 18 (3): 446-58.

[12] Maron, B. J. 2000. "The Paradox of Exercise." The New England Journal of Medicine 343 (19): 1409-11.

[13] Palhares, J. M., Benetti, M., Mazzei, L. C., and Bastos, F. C. 2012. "Profile and Preferences of practitioners of Street Racing: A Preliminary Study." Revista Mineira de Educação Física Special Edition: 1664-73. (in Portuguese)

[14] Hoffman, J. R., and Kaminsky, M. 2000. "Use of Performance Testing for Monitoring Overtraining in Elite Youth Basketball Players." Strength \& Conditioning Journal 22 (6): 54.

[15] Jürgens, D., Knechtle, B., Rüst, C. A., Knechtle, P., Rosemann, T., and Lepers, R. 2012. "An Analysis of Participation and Performance by Nationality at "Ironman Switzerland' from 1995 to 2011." Journal of Science and Cycling 1 (2): 10-20.

[16] Jokl, P., Sethi, P. M., and Cooper, A. J. 2004. "Master's Performance in the New York City Marathon 1983-1999." British Journal of Sports Medicine 38 (4): 408-12.

[17] Lee, D. C., Artero, E. G., Sui, X., and Blair, S. N. 2010. "Mortality Trends in the General Population: The Importance of Cardiorespiratory Fitness." Journal of Psychopharmacol 24 (4 Suppl): 27-35.

[18] Lepers, R., and Cattagni, T. 2012. "Do Older Athletes Reach Limits in Their Performance during Marathon Running?" Age (Dordr) 34 (3): 773-81.

[19] Leyk, D., Erley, O., Ridder, D., Leurs, M., Ruther, T., Wunderlich, M., Sievert, A., Baum, K., and Essfeld, D. 2007. "Age-Related Changes in Marathon and Half-Marathon Performances." International Journal of Sports Medicine 28 (6): 513-7.

[20] Nunomura, M. 2012. "Motives to Exercise Adherence related to the Variables Age, Gender, Education and Time of Permanence." Revista Brasileira de Atividade Física \& Saúde 3 (3): 45-58. (in Portuguese)

[21] Allender, S., Hutchinson, L., and Foster, C. 2008. "Life-Change Events and Participation in Physical Activity: A Systematic Review." Health Promotion International 23 (2): 160-72. 